\title{
El gran europeo Georg Friedrich Nicolai: médico y pacifista. Berlín, Alemania, 1874 - Santiago, Chile, 1964
}

\author{
FELIPE CABELLO C.
}

\section{The Great European Georg Friedrich Nicolai: physician and pacifist. Berlin, Germany, 1874 - Santiago, Chile, 1964}

Georg Friedrich Nicolai (1874-1964) was a German physician and physiologist whose pacifism during the First World War led him in 1914 to cosign with W. J. Foerster, A. Einstein and O. Bueck a "Manifesto to the Europeans" against the entry of Germany into the war and the invasion of Belgium. As a result of this appeal and his strong pacifism, Nicolai lost his positions as cardiologist to the German royal family, professor at the University of Berlin and chief of laboratory at the Charite hospital also in Berlin, and was sent as a garrison physician in Graundenz, in today's Poland. There he began to write his book, The Biology of War. It managed to avoid censorship and was published in Leipzig in 1916. He was court-martialed in Danzig in 1916 but escaped to Denmark. Nicolai was reinstated to his faculty positions by the Weimar Republic after the war but was subsequently forced to emigrate from Germany to South America by the pressure of right wing student groups who accused him of being a deserter and a traitor. From 1922 to 1932 Nicolai lived in Argentina, and from 1932 until his death in 1964, in Chile. In this later country Nicolai was professor in the University of Chile and interacted with members of the Chilean intelligentsia, including the poets Vicente Huidobro, Gonzalo Rojas and Pablo Neruda. Through his friendship with Chilean psychiatrist Agustin Tellez, Nicolai influenced the development of phenomenological psychiatric school in Chile. The Chilean novelist Fernando Alegría compared him favorably with Robert J. Oppenheimer and Linus Pauling.

(Rev Med Chile 2013; 141: 535-539).

Key words: Chile; History of Medicine; Politics; Science.

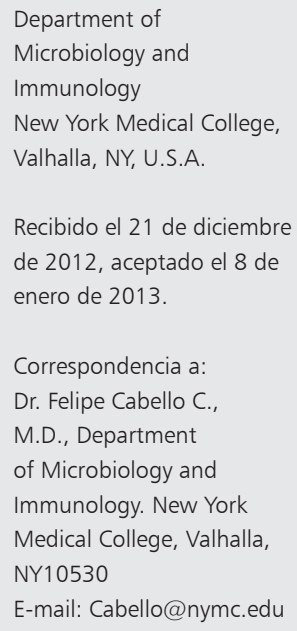

"Nuestro conocimiento aumenta más al tener una visión apasionada y valerosa de la realidad que al tener de esta una visión delicada y ecuánime, con apariencias de neutralidad; esta última nunca creará nada nuevo y original y sólo corregirá defectos".

G. F. Nicolai: "La biología de la guerra”, 1916.

\footnotetext{
A mediados de la década de 1980, en la búsqueda de información acerca de los problemas éticos generados en la práctica médica y en la salud pública en situaciones caracterizadas
}

por la ausencia de democracia política, como las que experimentaba Chile en esa época, encontré un artículo en Lancet del año 1986 relacionado con estas materias ${ }^{1}$. Su autor criticaba el servilismo de la profesión médica alemana hacia el nazismo, servilismo que con raras excepciones hiciera que se atropellaran los principios éticos más elementales de su actividad y que coronara en su entusiasta y criminal colaboración con el Holocausto y la eliminación física en masa de eslavos, disidentes políticos y enfermos mentales ${ }^{1}$. Al leer al autor dando las razones históricas que, según él, explicarían esta 
situación, algunas de las cuales se remontarían a la época de la Primera Guerra Mundial, encontré en su análisis el nombre del médico y científico Georg F. Nicolai ${ }^{1}$. Según el artículo, G.F. Nicolai, junto con A. Einstein, W.J. Foerster y O. Bueck, todos profesores de la Universidad de Berlín, habían sido los únicos intelectuales alemanes que se habían opuesto de manera pública en su "Llamado a los Europeos", a la participación de Alemania en la Primera Guerra Mundial, en 1914. Llamado que fue una respuesta al famoso "Manifiesto de los 93" que justificó la entrada de Alemania a la Guerra y la invasión de Bélgica ${ }^{1-4}$. El valor y la visión de Nicolai y de Einstein para oponerse al "Manifiesto de los 93" se aprecia al leer la lista de los firmantes de este último, ya que ellos representaban la flor y nata de la cultura e intelectualidad alemanas, destacándose entre ellos los médicos y científicos Röntgen, Waldeyer, von Behring, von Wasermann, Neisser, Ehrlich, Fisher, Haber, Otswald y Plank. La subordinación mayoritaria de la profesión médica alemana a los dictados del nazismo, desde 1933 a 1945, según el autor del artículo seria la continuación lógica del apoyo irrestricto de la mayoría de la intelectualidad alemana a la política belicista de Alemania desde la Primera Guerra Mundial ${ }^{1}$.

La lectura del nombre de G. F. Nicolai me trajo recuerdos de una clase de psiquiatría de comienzos de primavera en el Hospital Psiquiátrico de San-

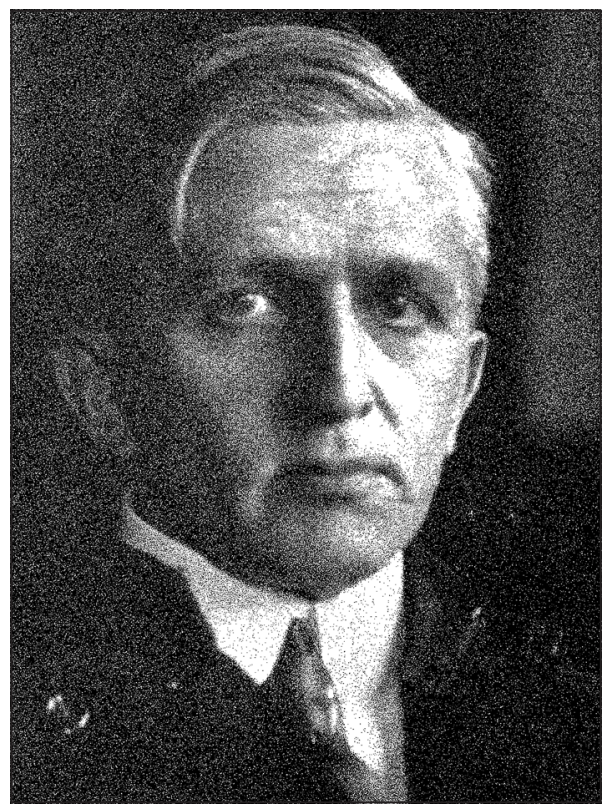

Figura 1. Georg Friedrich Nicolai. tiago, a mediados de la década de 1960. En aquella tarde nuestro profesor, Don Agustín Téllez M., nos preguntó con su característica parsimonia “¿Saben quién ha fallecido este fin de semana?” Ninguno de los estudiantes fue capaz de responder aquella enigmática pregunta y el profesor Téllez agregó, socarrón, algo así como que "Seguro que saben los más finos detalles de los partidos de football del sábado y del domingo pero desconocen al personaje importante para la medicina y la ciencia que ha fallecido en nuestro país este fin de semana". A continuación dijo: "Ha muerto el sabio Nicolai" y, sin más explicaciones, comenzó su clase. Como una persona absolutamente desinteresada en los deportes de espectadores, la antítesis establecida por el comentario del Dr. Téllez entre el sabio Nicolai y el football permaneció en mi memoria. A la luz de ese recuerdo, al leer el nombre de $\mathrm{Ni}$ colai y la descripción de sus actividades políticas junto a Einstein en el artículo del Lancet, se me despertó la curiosidad por saber si era la misma persona admirada por el Dr. Téllez ${ }^{1,2,5}$. G. F. Nicolai (Figura 1), antes de su colaboración política con Einstein, era un médico respetado y de gran reputación en Alemania antes de la Gran Guerra y como resultado de esto era cardiólogo privado de la familia real alemana y profesor titular de la Universidad de Berlin ${ }^{2}$. Nicolai, a quien se le adjudica ser uno de los pioneros de la electrocardiografía, había estudiado medicina en Könisberg (Prusia) y también había asistido a clases de medicina en Berlín, Heidelberg y París, recibiéndose de médico en $1899^{2}$. Además, había estudiado física con M. Plank, electrocardiografía y electrofisiología con Einthoven, en Leyden, y con Pavlov en San Petersburgo y llegó a ser director del laboratorio de investigaciones de la Segunda Clínica en el Hospital de La Charité, en Berlín, el mismo famoso hospital de su profesor Virchow y de Koch, Billroth y Creutzfeld, entre otros ${ }^{2,6}$.

Después de su colaboración con Einstein en contra de la entrada de Alemania a la guerra, la promisoria y ascendente carrera del Dr. Nicolai comenzó a deteriorarse y fue trasladado rápidamente en 1914 a un hospital provincial cerca de la fortaleza prusiana de Graudenz, ahora en Polonia, donde además de sus actividades clínicas escribió su famosa obra pacifista "La biología de la guerra", que fuera publicada a espaldas de la censura en Leipzig, en $1916^{2,7-9}$. Esta obra, fue positivamente criticada, entre otros, por los escritores y Premios 
Nobel franceses Romain Rolland (1915) y Anatole France (1921) e hizo que Nicolai fuera propuesto como candidato al Premio Nobel de la Paz, en $1919^{1,2,10}$. De acuerdo a Rolland, quien denominara a Nicolai como "El Gran Europeo", en esta erudita obra su autor ataca el patriotismo barato de los políticos y de sus seguidores, basados en falsos conceptos raciales y en la segunda parte de ella analiza las características de la sociedad alemana que favorecían esta irracional actitud belicista ${ }^{10}$.

Según Rolland, Nicolai adscribía parcialmente el belicismo alemán al fracaso de la filosofía de Kant en establecer un puente entre la razón pura y la razón práctica, lo que hacía posible que en nombre de los más altos ideales Alemania persiguiera políticas desastrosas y brutales, totalmente disociadas de ellos y las cuales alcanzaran su apogeo durante la Segunda Guerra Mundial ${ }^{2,10}$. Después de un intento para volver a la Universidad de Berlín en 1915-1916 y debido a que continuara con sus actividades pacifistas, Nicolai fue relegado a Danzing (Gdansk) donde fue sometido a una Corte Marcial días después de la publicación de su libro y condenado a seis meses de prisión ${ }^{2,8,9}$. Inmediatamente después de su salida de la prisión le fueron dadas órdenes de incorporarse al ejército, en 1918, lo cual de acuerdo a sus principios rehusó, escapando durante la noche en un avión militar a Dinamarca ${ }^{2}$. Una vez finalizada la guerra y caída la monarquía fue reincorporado por la República de Weimar como profesor en la Universidad de Berlín, en 1921. Sin embargo, y a pesar del apoyo que Einstein le prestara, las protestas de los estudiantes de derecha, ya en esa época organizados en grupos que adscribían la derrota alemana a la leyenda de "la puñalada en la espalda" al ejército alemán; leyenda que tan bien utilizara Hitler para ascender al poder, acusaban a Nicolai de ser un desertor y lo obligaron a renunciar y a emigrar a Sud América ${ }^{2,3}$.

Nicolai vivió desde 1922 por diez años en Argentina, primero en Córdoba y luego en Rosario y el año 1932 se trasladó a Santiago de Chile, donde vivió los últimos treinta y dos años de su vida hasta su fallecimiento a los noventa años de edad, en octubre de $1964^{2}$. En Chile, como lo manifestara el Profesor Agustín Téllez en un artículo de homenaje post mortem en esta Revista ${ }^{5}$, ejerció una importante influencia intelectual, siendo profesor de la Universidad de Chile en la Escuela de Medicina Veterinaria, en el antiguo Instituto
Pedagógico y en la Escuela de Medicina ${ }^{2.5}$. La biografía definitiva de Nicolai fue publicada, en inglés, en 1982 por el Dr. W. Zuelzer, director de la División de Hemopatías y Trasfusiones de los National Institutes of Health (EE.UU.) y compatriota de Nicolai. Esta biografía está basada en una gran cantidad de documentos archivados por él mismo y accesibles al autor después de su muerte ${ }^{2}$. Esta semblanza nos presenta a Nicolai como una persona de gran inteligencia, con un pensamiento eminentemente racional, enciclopédico y sintético y una gran facilidad para expresar y presentar sus ideas en forma clara y precisa ${ }^{2}$. Además de ser un hombre valeroso, intransigente en la defensa de sus principios, Nicolai fue un pensador independiente y de allí que fuera acusado en Chile, por ejemplo, de ser simultáneamente un "judío internacional que hace propaganda soviética" y de ser también un "reformista burgués más"2,11.

Esta biografía también nos revela aspectos obscuros de su compleja personalidad, como son el desapego a su familia, a sus hijos nacidos fuera del matrimonio y la instrumentalización de sus relaciones con algunas de sus innumerables amantes y varios de sus amigos ${ }^{2}$. El historiador atribuye la rebeldía y la originalidad de Nicolai a la cultura de un hogar inusual, formado por un padre judío que, al igual que Rudolf Virchow y el poeta Heinrich Heine, tomara parte activa en la revolución de 1848 en Alemania y fuera perseguido por esto, unido a una madre alemana, protestante y protofeminista, de una orgullosa familia de nobles terratenientes (Junkers) de Prusia oriental, emparentados con el compositor Otto Nicolai ${ }^{2}$. La influencia que George Nicolai tuviera sobre el ambiente intelectual, tanto en la medicina, la biología y las humanidades y que en el caso del profesor Téllez y la psiquiatría se manifestó a mi modo de ver en la lejanía que este último tenía por el psicoanálisis y por Freud, fue al parecer muy constante y extensa durante su permanencia en Chile $^{2,5,12}$. Además de clases impartidas en la Universidad, continuó escribiendo una serie de libros y artículos sobre aspectos filosóficos e históricos de la biología y de la medicina y dando conferencias fuera de la Universidad y a través del país sobre los mismos temas ${ }^{2,5,11}$.

Su influencia también se aprecia al leer textos, entrevistas, comentarios y correspondencia encomiástica de los literatos chilenos que lo conocieron, como el poeta Vicente Huidobro, con el que 
participara en el círculo literario "Mandrágora"13, el recientemente fallecido poeta laureado Gonzalo Rojas $^{14}$, el escritor y político Volodia Teitelboim, el poeta Pablo Neruda y el novelista Fernando Alegría, quien al parecer fuera su alumno en el Pedagógico ${ }^{15,16}$. Este último, con quien yo compartiera socialmente durante mi estadía en la Universidad de Stanford donde él era profesor y a quien conocí como un hombre sobrio, escribe sin embargo, entusiasmadamente sobre Nicolai diciendo que su personalidad lo impresionó tanto o más que las personalidades de los científicos estadounidenses Robert J. Oppenheimer y Linus Pauling, a quienes Alegría conociera en Berkeley y en Stanford, respectivamente ${ }^{16}$. Es interesante que estos dos científicos, al igual que Nicolai, además de ser amigos de Einstein fueron pacifistas y similarmente a él fueron perseguidos por su gobierno. El pacifismo de Pauling se manifestó entre otras por ser miembro junto con Einstein del Comité de Emergencia de Científicos Atómicos en 1946 y en la firma de la declaración Russell-Einstein en 1955, ambas actividades en contra de las armas nucleares. El pacifismo de Oppenheimer comenzó después que dirigiera el "Proyecto Manhattan" que desarrollara la bomba atómica, con su oposición basada en fundamentos éticos al desarrollo de la bomba de hidrógeno, en 1947.

Sin lugar a dudas que como Romain Rolland lo dijera, Nicolai, además de ser un precursor en variados aspectos de la medicina, fue también un precursor de los movimientos pacifistas en el mundo y probablemente su emigración a Argentina y a Chile y el advenimiento del nazismo en Alemania impidieron que sus esfuerzos alcanzaran resonancia mundial ${ }^{2}$. Él es también un ejemplo de aquellos intelectuales "engagee" como los llamara Jean Paul Sartre, que sin ambages enfrentan los desafíos éticos impuestos por los desbordes del poder político a pesar de que en este enfrentamiento arriesguen su posición social, su libertad y aún sus vidas. La biografía de Nicolai describe en forma detallada su vida en Alemania, pero aspectos de sus cuarenta años de vida en Argentina y Chile son descritos con menor detalle y están a la espera de un historiador que los analice, por su interés, como esperamos haber demostrado en este ensayo.

Agradecimientos: Dedico este artículo a la memoria de mi amigo y colega, el profesor Félix Wassermann y de su esposa Hanna . F. Wassermann nacido en Alemania y sobrino del conde Dr. August Von Wassermann, creador de la reacción serológica del mismo nombre y desgraciadamente signatario del "Manifiesto de los 93" en 1914, me honró con su amistad por más de treinta años durante los cuales me introdujo a varios aspectos de la cultura alemana y de la historia de la microbiología en ese país. Además de entretenerme a menudo con la narración irónica de las peripecias de su prolongada escapada de Alemania a fines de 1930 a los Estados Unidos de Norteamérica vía Inglaterra, Cuba y México y del servilismo de sus perseguidores al verlo volver a su ciudad natal, Bamberg, como miembro del ejército estadounidense, en 1945. Agradezco al profesor Agustín Téllez M. la introducción a G.F. Nicolai y la enseñanza de una psiquiatría racional alejada del psicoanálisis. Agradezco al escritor Juan A. Epple, profesor del Department of Romance Languages, Universidad de Oregon, Portland, la cita bibliográfica de Fernando Alegría en que se menciona a G.F. Nicolai. La Dra. Katia Velásquez aportó sugerencias al texto y la Srta. Jessica Gallardo, de la Biblioteca de la Facultad de Medicina de la Universidad de Chile me facilitó acceso a material bibliográfico referente a G. F. Nicolai.

\section{Referencias}

1. Hanauske-Abel HM. From Nazi Holocaust to nuclear holocaust: A lesson to learn. Lancet 1986; 2: 271-3.

2. Zuelzer W. The Nicolai Case.Wayne State University Press, 1982. Detroit, MI.

3. Rowe DE, Schulmann R. Einstein on Politics: His Private Thoughts and Public Stands on Nationalism, Zionism, War, Peace and the Bomb. Princeton University Press, 2007. Princeton, NJ.

4. Sri Kantha S. Einstein's medical friends and their influence on his life. Medical Hypotheses 1996; 46: 257-60.

5. Téllez A. Vida, obras e ideas del Profesor Nicolai. Rev Med Chile 1965; 93: 727-31.

6. Kastner I. The German physician and pacifist Georg Friedrich Nicolai (1874-1964) as a pupil of the Russian physiologist Ivan Petrovic Pavlov (1849-1936). [Article in German]. Wurzbg Medizinhist Mitt 2005; 24: 261-7.

7. World War I. Nov 16, 1914: New Fatherland League launched in Germany. http://www.history.com/this-dayin-history/new-fatherland-league-launched-in-germany

8. Grande J. German Author Condemns War. Dr. Nicolai of Berlin University assails it as a biological anomaly. 
Jailed for his opinions. The New York Times, November 20, 1917.

9. The New York Times. Truth about Dr. Nicolai. German Professor jailed despite Berlin's official denials. February 10, 1918.

10. Rolland R. The Forerunners. Harcourt, Brace and Howe, 1920. New York.

11. Jorge Federico Nicolai. Sesión ordinaria del 9 de octubre de 1934. Arch Soc. Cir Hosp. 1934; 4: 337.

12. Baeza GAM. El Profesor Agustín Téllez Meneses. Reseña de su obra y personalidad (Influencia de la Psiquiatría alemana en la chilena). Rev Psiquiatría 1990; 7: 369-74.

13. Lizama P. Huidobro y la vanguardia de los años 30. 2008. http://vicentehuidobro.uchile.cl/ensayo_patricio_lizama.

14. Guerrero PP. Gonzalo Rojas en su laberinto. 2007. http:// revistaliterariaazularte.blogspot.com/2007/10/pedropablo-guerrero-gonzalo-rojas-en.html

15. De Mussy Roa LG. Mandrágora: la raíz de la protesta o el refugio inconcluso. 2012. http://www.mandragora. uchile.cl/critica/testimonios/grupal/3.htm

16. Alegría F. 1983. Una especie de memoria. Editorial Nueva Imagen. Caracas, pp. 134-37. 